\title{
La comunicación y la asertividad del discurso durante las interacciones grupales presenciales y por computadora
}

\author{
Nancy Noemi Terroni ${ }^{1}$ - Universidad Nacional de Mar del Plata, Mar del Plata, Argentina
}

\begin{abstract}
Resumen
En este trabajo se reportan los resultados del análisis reticular en la comunicación, y los puntajes de la asertividad del discurso de los partipantes de pequeños grupos que resuelven una tarea de recuperación de memoria (La guerra de los fantasmas, Bartlett, 1932). El diseño es cuasiexperimental y los 90 participantes alumnos de la Universidad Nacional de Mar del Plata, debieron reconstruir la misma en forma grupal colaborativa. Los sujetos fueron asignados aleatoriamente a los grupos y a las dos condiciones (grupos cara a cara y mediados por computadora). Se registraron las interacciones presenciales mediante video filmaciones y las electrónicas quedaron almacenadas en el canal de chat. En general, la asertividad del discurso y la comunicación presentaron asociaciones significativas, con algunas diferencias según el canal comunicacional empleado. Se discuten estos resultados con relación al tipo de tarea y a las restricciones de los medios electrónicos.

Palabras-clave: comunicación; asertividad; Grupos; influencia; computadora.
\end{abstract}

\section{Communication and assertiveness on the discourse during face-to-face and computer-mediated group interactions}

\begin{abstract}
This work reports the results of reticular analysis in communication, and the scores from the discourse assertiveness of the participants of small groups who solve a recall memory task (The war of the ghosts, Bartlett, 1932). The design is a quasi-experimental one and the 90 subjects, students from Mar del Plata University had to reconstruct the same story in collaborative groups. The subjects were assigned in an aleatory way, to both conditions (face to face and computer-mediated groups). The subjects' interactions in face-to-face communication groups were recorded in video films and the electronic ones were stored in the chat channel. In general, the discourse assertiveness and the communication presented significant associations, with some differences according to the communication channel used. These results are discussed about the type of task and the restrictions of the electronic media.
\end{abstract}

Keywords: communication; assertiveness; groups; influence; computer.

\section{Introducción}

Se han llevado a cabo innumerables investigaciones destinadas a examinar la capacidad que poseen los sujetos a la hora de solucionar problemas interpersonales, ya sea en situaciones ecológicas o cuasi experimentales (éstas últimas bajo un riguroso control de variables). La cantidad y tipo de emisiones efectuadas en situaciones grupales también ha sido objeto de estudios, relacionando dichos resultados con la influencia o procesos de liderazgo que se producen a nivel grupal.

En este estudio se analizan las interacciones que se producen en los pequeños grupos presenciales (CAC) y mediados por computadoras a través del canal de chat (CMC) a la hora de resolver una tarea de tipo intelectiva, más precisamente un reactivo de recuperación colectiva de memoria.

Para ello se cuenta con el registro de las

${ }^{1}$ Endereço para correspondência:

San Juan, 508 (C.P. 7600). Mar del Plata, Argentina.

E-mail: nterroni@mdp.edu.ar dinámicas grupales, y una serie de evaluaciones y autorreportes (percepción de influencia y asertividad del discurso), medidas éstas que, en muchos casos fueron sometidos a evaluación interjueces para una clasificación objetiva.

\section{Antecedentes previos}

La revisión histórica nos revela los primeros trabajos que identifican y conceptualizan los elementos constitutivos del acto comunicativo (Jakobson, 1963; Shannon \& Weaver, 1949; Watzlawick, Bavelas \& Jackson, 1997): emisor, receptor, canal, mensaje y código empleado.

Trabajos pioneros como los realizados en torno a las interacciones de los pequeños grupos realizados por Bales y colaboradores a lo largo de la década de 50-70 han constituido un impulso sistemático en estudios sobre la dinámica grupal. $\mathrm{Su}$ modelo del equilibrio analiza las fuerzas internas a la dinámica grupal, más que las presiones externas al grupo y, por lo tanto, su teoría llega a ser conocida como teoría de la interacción. En primer lugar, estos autores construyeron un esquema observacional para codificar las conductas grupales, conocido como 
Análisis del Proceso de Interacción, IPA (Bales \& Strodbeck, 1951) y luego generaron un modelo ampliado denominado Sistema para el Múltiple Nivel de Observación de Grupos denominado SYMLOG (Bales \& Cohen, 1979). Dichos trabajos constituyen la base de desarrollos posteriores y actuales, como los efectuados por Emitt y Gorse (2006) quienes analizan la relación existente entre los resultados de los proyectos y las comunicaciones de los equipos de trabajo en ámbitos organizacionales. A lo largo del examen minucioso de las emisiones e interacciones de dichos equipos, según la clasificación IPA de Bales, estos investigadores detectaron la existencia de patrones comunicacionales de interacción que se asociaban con los resultados (exitosos o desfavorables) de los proyectos de grupo.

Retrocediendo en la línea histórica, cabe citar otros estudios (Carter \& cols., 1951) que también constituyeron la base del análisis funcional de la interacción grupal. Diferentes investigadores de la comunicación (Fisher, 1970; Gouran, 1969; Gouran \& Hirokawa, 1983; Mabry, 1975) han considerado que intervienen funciones del procesamiento social de la información, las cuales involucran el análisis y la combinación de la información, así como la generación, la elaboración y la evaluación de las ideas; funciones analíticas que involucran el análisis de las circunstancias y las contingencias que rodean a la situación de toma de elección; funciones procedurales, abarcando el establecimiento y mantenimiento de procedimientos y reglas para llegar a la decisión; funciones vinculadas a los objetivos, las que pertenecen al establecimiento y monitoreo de los objetivos grupales y valores; funciones sinergéticas, que abarcan la coordinación y la motivación de los miembros del grupo y, finalmente, las funciones retóricas, tales como la persuasión, la influencia social, el liderazgo y la visión. Resulta indiscutible que la comunicación constituye el elemento fundamental de las interacciones y por ende de la naturaleza humana.

La comunicación se distingue por ser un proceso multifacético, que ocurre al mismo tiempo en varios niveles - consciente, subconsciente $\mathrm{e}$ inconsciente - y como parte orgánica del dinámico proceso de la vida misma (Bordenave, 1985). Las definiciones en general apuntan cada vez más a los procesos de intercambio entre emisor y destinatario dentro de una interacción múltiple, y enfatizan la importancia del contexto social en el que se da esa comunicación, así como sus dimensiones estructurales y procedurales; en desmedro de la visión lineal y unidireccional mecanicista que en alguna oportunidad prevaleciera.

Otro rasgo importante de la comunicación, es la asertividad. Este factor juega un papel importante en las interacciones grupales, ya que constituye una habilidad o destreza a la hora de emitir opiniones y en los procesos de influencia grupal.

La palabra asertivo proviene del Latin (asertus) y significa afirmar con certeza alguna cosa, y por lo tanto se considera que la persona asertiva es aquella que puede enunciar con certeza sus opiniones y deseos.

Realizando un rastreo histórico, los investigadores españoles Güell y Muñoz (2000) comentan que el concepto de conducta asertiva fue empleado por primera vez por Wolpe en 1958 y posteriormente ha tenido muchas elaboraciones. Por ejemplo, en el campo de las habilidades sociales han trabajado autores como Hersen y Bellack (1977), mientras que los aspectos concernientes al entrenamiento asertivo han sido tratados inicialmente por Alberti y Emmons (1978) con la consecuente elaboración de diversos registros e inventarios (Rathus 1973; Wolpe \& Lázarus, 1966).

Existen definiciones que resaltan el contenido de la conducta (opiniones, sentimientos, deseos, etc.) y otras las consecuencias o resultados. Entre las primeras, diversos autores definen al individuo asertivo como aquella persona que tiene una personalidad excitativa o activa, el que define sus propios derechos y no presenta temores en su comportamiento (Fensterheim \& Baer, 1976). Estos autores además caracterizan a la persona asertiva por presentar: a) libertad de expresión; b) comunicación directa, adecuada, abierta y franca; c) facilidad de comunicación con toda clase de personas $y$ d) un comportamiento respetable aceptando las limitaciones.

Para Carrobles (1979), la asertividad es la habilidad para exponer de manera apropiada y directa, en un momento determinado (de índole personal o social), las creencias y sensaciones tanto positivas como negativas. Navarro Rodríguez (2000) lo define como un conjunto de principios y 
derechos que hacen a un modelo de vida personal enfocado a lograr el éxito en la comunicación humana. Su esencia radica en la habilidad para transmitir y recibir mensajes haciéndolo de forma honesta, respetuosa y oportuna. Posee relación directa con el saber decir, con el control emocional y con el lenguaje corporal. Por lo tanto, en la asertividad intervienen variables comunicacionales linguísticas así como otros factores paralinguísticos (contacto visual, gestos, entonación, etc.).

En tanto que, para varios autores la conducta asertiva debería definirse en términos de la eficacia en una situación, más que en términos de su topografía (Argyle, 1981, 1984; Kelly, 1982; Linehan, 1984), si bien resulta controvertido pensar una conducta en términos de sus resultados según otros investigadores (Arkowitz, 1981; Shroeder \& Rakos, 1983).

Caballo (1993) considera como equivalentes la denominación de "asertividad" o "habilidades sociales" y por ende al "entrenamiento asertivo" o "entrenamiento en habilidades sociales". En tanto, otros autores manifiestan que la noción habilidades sociales resulta más extensa y no se agota en la asertividad (Del Prette \& Del Prette, 1999; Falcone, 1989, 1995, 1998; MacKay, 1988; Matos, 1997). Una revisión de estudios efectuados por Falcone (1998, 2000) sugieren que la empatía también es una habilidad social, y que en algunos contextos resulta más adecuada que el término asertividad. Aun cuando la asertividad y la empatía se complementan en varias situaciones sociales.

La definición propuesta por Caballo (1986) auna ambos aspectos de la conducta asertiva (tanto el proceso como el resultado de la acción):

la conducta socialmente habilidosa es ese conjunto de conductas emitidas por un individuo en un contexto interpersonal que expresa los sentimientos, actitudes, deseos, opiniones o derechos de ese individuo de un modo adecuado a la situación, respetando esas conductas en los demás, y que generalmente resuelve los problemas inmediatos de la situación mientras minimiza la probabilidad de futuros problemas.

En esta clasificación entran las siguientes capacidades (Caballo, 1991): iniciar y mantener conversaciones; hablar en grupo; expresar amor, afecto o desagrado; defender los propios derechos; solicitar favores; rechazar pedidos; hacer y aceptar cumplidos; expresar las opiniones (acuerdos o desacuerdos); manifestar justificadamente cuando la persona se siente molesta, enfadada; saber dar disculpas o reconocer el desconocimiento; saber enfrentar las críticas y solicitar modificaciones en el comportamiento a otro.

En Brasil, Del Prette e Del Prette (2001) presentan una taxonomía más completa que la desarrollada por Caballo (1991) al considerar dimensiones más amplias: 1. Habilidades sociales de comunicación (hacer y responder preguntas, elogiar y gratificar; pedir feedback, etc.); 2. Habilidades sociales ciudadanas (agradecer, pedir por favor, etc.); 3. Habilidades sociales asertivas de enfrentamiento (opinar en discordancia, concordancia; aceptar o rechazar pedidos; disculparse, admitir fallas, etc.); 4. Habilidades sociales hepáticas (parafrasear, expresar apoyo, etc.); 5. Habilidades sociales de trabajo (hablar en público; organizar un grupo; tomar decisiones o mediar conflictos, etc.); 6. Habilidades sociales para expresar sentimientos positivos (expresar solidaridad; hacer amigos, cultivar amor, etc.). Además de los componentes verbales, Del Prette \& Del Prette (1999) consideran los componentes no verbales de la comunicación: expresión facial, postura corporal, gestos, movimientos de cabeza, entre otros.

$Y$ de las numerosas investigaciones desarrolladas en ese país en torno a la asertividad o habilidades sociales, se pueden mencionar las de Furtado, Falcone y Clark (2003) quienes evaluaron la correspondencia entre los niveles de estrés y las habilidades sociales en los estudiantes de medicina de una universidad de Rio de Janeiro, hallando relaciones significativas $y$ diferencias entre géneros. En otra investigación (Del Prette \& cols., 2004) los científicos evaluaron las habilidades sociales de los estudiantes de Psicología, que concurrían a 4 Universidades de Brasil $(\mathrm{N}=564)$, quienes completaron un inventario (IHS-delPrette) en torno a cinco factores. Se compararon los resultados según el lugar de estudio, el género y la edad, encontrando diferencias significativas en algunas medidas.

Cabe citar además, un estudio que apunta a medir las habilidades sociales en la tercera edad (Carneiro \& Falcone, 2004). Estos investigadores entrevistaron a $\mathrm{N}=30$ estudiantes de la Universidad Abierta de la Tercera Edad. Se 
examinaron diversas situaciones sociales a través de una entrevista que constaba de 32 ítems basada en estudios previos (Falcone \& cols., 2001); los resultados exhibieron que los adultos mayores presentaban deficiencias en algunas de las situaciones sociales, por lo ese estudio plantea la posibilidad de efectuar programas de entrenamiento.

Finalmente, haciendo una breve mención a los medios electrónicos como dispositivos que se implementan en las interacciones sociales, y por ende modulan las comunicaciones y la asertividad del discurso, la revisión de trabajos científicos previos exhibe que el uso de las Nuevas Tecnologías de la Información y la Comunicación (NTIC) desarrolla un lenguaje desinhibido, gestión negativa de conflicto, dificultades en la coordinación y la retroalimentación, problemas para alcanzar consenso, polarización del grupo y dificultades para la percepción de la influencia en el grupo (Goode \& Johnson, 1991; Harasim \& Winkelmans, 1990; Hiltz, Johnson \& Turoff, 1986; Hirokawa \& Poole, 1996; Kiesler, Siegel \& McGuire, 1984; Peiró, Prieto \& Zornoza, 1993; Terroni, 2001; Vivas \& Terroni, 2001). Otras investigaciones analizan las asociaciones entre las comunicaciones y la influencia en pequeños grupos intelectivos, así como las cantidades de alocuciones de uno y otro tipo según el canal comunicación al (cara a cara y mediado por computadora). En línea general se hallaron asociaciones significativas entre la centralidad comunicacional y la influencia grupal; también entre la centralidad comunicacional y la asertividad, aunque se exhibieron diferencias entre las proporciones de uno y otro tipo según las restricciones del medio (Terroni, 2003a; 2003b, 2005, 2006, 2007)

En el presente trabajo se reportan los resultados de emplear medidas provenientes del análisis de redes sociales para evaluar los volúmenes y direccionalidad de las comunicaciones grupales; también se examinan los puntajes de la asertividad de los diálogos y la percepción de influencia y la influencia ejercida por los participantes durante la dinámica grupal. Se trata de pequeños grupos de resolución de tareas, integrados por cinco participantes cada uno, quienes debieron resolver un reactivo de recuperación de memoria en dos medios: cara a cara o mediados por computadora a través del canal de chat.

\section{Método}

\section{Participantes}

La muestra está conformada por 90 alumnos de 3er. año de Psicología, cursantes de la asignatura Psicología Cognitiva. Para la condición mediada por computadora (CMC) se seleccionaron 45 alumnos y para la condición presencial (CAC) se seleccionaron otros 45 alumnos. Los grupos se componen de 5 miembros cada uno seleccionados en forma aleatoria en todos los casos.

\section{Materiales}

Se utilizó como reactivo de recuperación de memoria, la historia "La guerra de los fantasmas" (Bartlett, 1932). Es una tarea de tipo intelectiva, de acuerdo a los cuatro criterios de demostrabilidad de Laughlin y Ellis (1986), que permite discriminar mejor la relevancia de la capacidad de recuperación de memoria de los participantes, así como los procesos de argumentación en la influencia social en el grupo. El puntaje se obtuvo evaluando las unidades recuperadas según la valoración otorgada (Mandler \& Johnson, 1977). De acuerdo a la misma, se identificaron 42 unidades de contenido, y las mismas fueron puntuadas 0 si la unidad no era recordada, 0,50 si la unidad era recuperada en forma parcial y 1 punto si la unidad era recordada en su totalidad.

Diseño

Es similar para ambas modalidades, cara a cara y mediado por computadora. Los participantes escuchan dos veces una cinta audiograbada conteniendo la historia a recuperar. Luego se les entrega a cada uno de los sujetos una tarea distractora de laberintos de modo tal de vaciar la memoria corto plazo y a posteriori recuperar la historia en forma individual. Tras esta primera fase de recuerdo individual, se conforman al azar grupos de 5 miembros cada uno, quienes, en forma cooperativa, deben reconstruir la historia lo más fidedignamente posible consensuando una historia grupal común. En una tercera fase, se le solicita a los participantes nuevamente el recuerdo individual de la historia, es decir la reconstrucción definitiva de la historia. Finalmente, se administra a los participantes una escala de tipo likert 
explorando la influencia percibida durante la interacción grupal.

En la modalidad mediada por computadora, los grupos de cinco participantes interactuaron de manera electrónica a través de un canal privado de chat, con cinco máquinas horizontalmente interconectadas. Los participantes podían enviar y recibir mensajes en forma conjunta con los otros cuatro miembros del grupo o en forma privada a un interlocutor en particular.

Tanto para este registro, como para la clasificación de las unidades comunicativas según el contenido, se tomaron en cuenta las video filmaciones de las interacciones cara a cara y lo almacenado en los registros del log del chat, llevándose a cabo una evaluación interjueces de las categorías propuestas. Una vez efectuado esto, se obtuvo una clasificación definitiva de la comunicación de cada uno de los integrantes del grupo durante la tarea. El mismo procedimiento correspondió a la asertividad del discurso, la cual se sometió a una evaluación interjueces de acuerdo con una escala likert de 5 puntos.

En el procedimiento se contemplaron consignas individuales y grupales. Las mismas estaban redactadas y fueron leidas por los investigadores para evitar sesgos en la forma de presentar las modalidades de resolución. Además, la segunda consigna grupal fue establecida para que los grupos se comporten de la forma más colaborativa o cooperativa posible, evitando que miembros del grupo impongan sus opiniones sin una debida fundamentación o discusión. La tercer consigna resulta adecuada para que los sujetos puedan resolver en forma autónoma la tarea expresando su última respuesta posible.

Consigna 1, Individual. "En forma individual Ud. deberá reconstruir la historia previamente escuchada de la manera más fidedigna posible". Una vez finalizada esta tarea, se entrega a cada uno de los participantes una serie de laberintos, la tarea distractora, con la consigna: "Ahora Ud. deberá resolver en forma individual una serie de laberintos que le serán entregados".

Consigna 2, Grupal. "Ahora en forma grupal Uds. deberán reconstruir una vez más la historia previamente escuchada de la manera más fidedigna posible". Por lo que el grupo deberá ponerse de acuerdo y consensuar una única historia, respetando las siguientes reglas: (1) Evite imponer a los otros su decisión personal; argumente con lógica; (2) Evite ceder sólo para obtener la unanimidad o para eludir conflictos; apoye otros puntos de vista sólo si usted está de acuerdo con ellos al menos en parte; (3) Evite técnicas de solución de conflictos como, por ejemplo elección de la mayoría, cálculo del término medio o transacción; (4) Considere las opiniones discrepantes como contribuciones útiles, en vez de sentirlas como perturbadoras".

Consigna 3, Individual. "Ud. ha recuperado la historia en forma individual y luego de modo grupal llegando a una única reconstrucción de la historia en forma consensuada. Probablemente algunos de sus recuerdos han sido modificados por el grupo y, recíprocamente, sus recuerdos habrán influenciado los de otros participantes. Le solicitamos que ahora nuevamente en forma individual nos presente la historia que para $\mathrm{Ud}$. represente la reconstrucción más fidedigna del original".

Consigna 4 (para evaluar la percepción de influencia una vez que la tarea se llevó a cabo). En una escala de cinco intervalos entre mucho y nada, cada participante deberá evaluar la siguiente cuestión "En tu opinión que influencia ha ejercido cada uno de los miembros del grupo sobre las decisiones que se han tomado" (Zornoza \& cols., 1993).

Definición conceptual y operacional de las variables

1)Unidades Comunicativas. Se consideran como intervenciones a toda expresión que produjeran los sujetos y que haya quedado registrada en la audiograbación o en el log. del chat. Dentro de las intervenciones puede haber una o más unidades comunicativas, definiendo a las mismas como toda intervención o fragmento de intervención emitido durante el proceso de interacción y que tenga sentido y significado en sî misma. Tomando como base una clasificación pionera (Bales \& Strodbeck, 1951) así como una categorización propuesta más recientemente (Roselli, Bruno \& Evangelista, 2000), es que integrantes del grupo de investigación efectuaron una grilla clasificatoria de las comunicaciones (Ehuletche \& Terroni, 2002), a la que luego se le agregó una clase adicional de comunicaciones, pertenecientes a las alocuciones de índole desinhibidas o flaming (Terroni, 2008 en prensa), en concordancia con los estudios los pioneros que analizaron dicho fenómeno (Kiesler, Siegel \& Mc 
Guire, 1984; Peiró, Prieto \& Zornoza, 1993; Siegel, \& cols., 1986).

De acuerdo con la clasificación de las comunicaciones emitidas durante las interacciones grupales, dentro de las Unidades Comunicativas de tipo Cognitivas se diferencian:

a. Unidades Comunicativas Conceptuales. Cuando los contenidos se relacionan con la tarea y son unidades de recuerdo, argumentaciones, contraargumentaciones, pedidos de información, asesoramiento, etc. Ejemplos de esta categoría. "Dos hombres bajaron al río a cazar focas"; "Si después sigue se escucharon ruidos de guerra"; "Yo recuerdo la parte que dice... y entonces dijeron que no tenian flechas, lo otro no lo recuerdo"; "creo que nos olvidamos de algo".

b. Unidades Comunicativas de Gestión de la Tarea. Cuando impliquen aportes para lograr el consenso de los miembros del grupo para realizar algo de una determinada manera, sirven para organizar y ordenar las interacciones del grupo. Ejemplos de esta categoría: "Bueno, entonces sigamos"; "Contemos lo que nos acordamos"; "Si les parece bien que la 1 ordene".

c. Unidades Comunicativas Socioemocionales (SE). Son aquellas unidades comunicativas que aporten sentimientos, emociones, valores, estados de ánimo, etc. Ejemplos de esta categoría, ambas tareas: "Esto fue todo un éxito"(S); "Jajaja" (S); "(Risas)" (S).

d. Comunicación Desinhibida (D). Se agrega esta categoría, tomando en consideración la denominada comunicación desinhibida o flaming (Siegel et. al., 1986). Incluye insultos, expresiones agresivas y lenguaje informal. Ejemplos de esta categoría: "Me fui al carajo, no?" $(D)$; "Esto es un quilombo" (D); "“Qué onda???" (D).

2) Asertividad del Discurso. En general los autores coinciden en señalar como característica principal de la asertividad de la comunicación, a la habilidad para exponer en determinado momento, de manera apropiada y directa creencias y sensaciones, positivas o negativas; considerando que cuando un individuo es asertivo se exhibe como expresivo, espontáneo, seguro y con mayor capacidad de influenciar a los otros (Alberti, 1977; Alberti \& Emmons, 1978; Carrobles, 1979). Se combinan para ello componentes verbales y no verbales de la manera más efectiva posible de modo tal que un sujeto en una situación de interacción grupal se manifiesta como eficaz y con conductas estratégicas, ubicándose esta conducta en una posición intermedia de acuerdo a un continuo que va desde la sumisión a la agresividad. Con la finalidad de evaluar la asertividad del discurso (con indicadores tales como: la claridad del discurso, tono de voz, seguridad, etc.) durante el proceso grupal, se examinan las hojas con las desgrabaciones de las interacciones presenciales (CAC) y los logs provenientes del trabajo mediado por computadora (CMC) otorgando un puntaje a cada participante (de $0=$ nada asertivo a $5=$ muy asertivo), el cual es sometido a una evaluación interjueces.

3) Centralidad Comunicativa. Según el Análisis de Redes, el grado de Centralidad (Freeman, 1979) es la medida que caracteriza a un miembro de la red en función de la cantidad y fortaleza de los vínculos que emite (grado normalizado de salida). El prestigio es la medida complementaria que se basa en las recepciones (grado normalizado de entrada). Ambas medidas son indicadores de prominencia o actividad en el grupo (visibilidad en la red social) y por ende, de la capacidad de influencia en el grupo. Se considera que el sujeto con mayor prominencia o actividad grupal (quien monopoliza las discusiones) será quien posiblemente ejerza más influencia. La centralidad se mide de acuerdo al número de intervenciones o emisiones de cada sujeto durante la fase grupal, y se emplea el programa de análisis de redes (Ucinet 5) el cual arroja para cada participante un valor del grado de centralidad de ese sujeto en la red (Borgatti, Everett \& Freeman, 1999).

4) Influencia Efectiva. La influencia real ejercida por cada participante en la tarea grupal conforma un puntaje de contribución al producto grupal (CPG) según la operacionalización efectuada por autores españoles (Zornoza \& cols., 1993). Dicho puntaje resulta de la sumatoria $(\Sigma)$ de las diferencias entre el recuerdo individual y el recuerdo grupal. El mayor puntaje en términos de concordancia, está en consonancia con la mayor influencia a nivel grupal.

5) Influencia percibida. Es la contribución al producto grupal percibida por cada uno de los integrantes del grupo (Zornoza \& cols., 1993). Para ello se emplea un inventario con una escala de tipo likert de puntuación de $1=$ poca contribución a $5=$ mucha contribución al producto grupal. Se suman los puntajes otorgados por cada uno de los sujetos incluido el mismo participante, pudiendo variar el puntaje total de 5 (mínimo) a 25 (máxima percepción de influencia). Esta 
constituye una medida subjetiva de influencia, la cual puede complementarse con medidas objetivas de influencia como la divergencia de opiniones individual- grupal o contribución real al producto grupal y las provenientes del análisis de redes (centralidad, prestigio, influencia y densidad)

\section{Resultados}

Tanto para la centralidad de la comunicación (total, conceptual, gestión de tarea y socioemocional) como para la asertividad del discurso, por ser variables no paramétricas, se empleará el análisis ro de Spearman para corroborar la posible asociación estadística entre dichas variables.

En los grupos presenciales (CAC) se puede observar la asociación existente entre la asertividad y la centralidad comunicacional: comunicación total $(r=0,77, \quad p<0,01)$, con la comunicación tendiente a organizar o gestionar el grupo $(r=0,66, p<0,01)$ y con la comunicación conceptual $(r=0,73, \quad p<0,01)$. Un análisis más exhaustivo al interior de esta última clase, muestra que las unidades comunicativas novedosas se asocian con la asertividad $(r=0,58, p<0,01)$ así como las alocuciones no novedosas $(r=0,70$, $p<0,01)$. También presentó asociaciones significativas la asertividad del discurso con las emisiones socioemocionales, si bien dichas asociaciones fueron más débiles $(r=0,34, p<0,05)$. Con el único tipo de comunicación con el cual la asertividad no presentó asociaciones significativas, es con la comunicación desinhibida $(p>0,05)$.

$\mathrm{Al}$ analizar la comunicación y la asertividad con la influencia ejercida por los miembros del grupo y la percepción de influencia, en línea general la comunicación presentó asociaciones significativas con la influencia (objetiva y subjetiva). En tanto la asertividad presentó asociaciones significativas únicamente con el aspecto subjetivo de la influencia (es decir, con la percepción de influencia, $p<0,05)$ (Ver Tabla 1).

Tabla 1. Correlación ro de Spearman entre los tipos de comunicación, la asertividad del discurso y la influencia (real y percibida) en grupos CAC. Tarea de Recuperación de Memoria.

\begin{tabular}{lllllllllll}
\hline & CTOT & GT & CONC & EMOC & CN & CNN & DESIN & ASERT & ICPG & PINFL \\
\hline CTOT & 1,000 & $0,828^{* *}$ & $0,963^{* *}$ & $0,546^{* *}$ & $0,451^{* *}$ & $0,951^{* *}$ & 0,233 & $0,770^{* *}$ & $0,332^{* *}$ & $0,506^{* *}$ \\
& & 0,000 & 0,000 & 0,000 & 0,003 & 0,000 & 0,089 & 0,000 & 0,003 & 0,000 \\
GT & 1,000 & $0,697^{* *}$ & $0,353^{*}$ & 0,130 & $0,671^{* *}$ & 0,051 & $0,663^{* *}$ & $0,346^{*}$ & $0,319^{*}$ \\
& & & 0,000 & 0,019 & 0,228 & 0,000 & 0,386 & 0,000 & 0,021 & 0,031 \\
CONC & & & 1,000 & $0,547^{* *}$ & $0,532^{* *}$ & $0,982^{* *}$ & 0,243 & $0,739^{* *}$ & $0,386^{*}$ & $0,525^{* *}$ \\
& & & & 0,000 & 0,001 & 0,000 & 0,080 & 0,000 & 0,011 & 0,001 \\
EMOC & & & 1,000 & 0,264 & $0,549^{* *}$ & $0,401^{* *}$ & $0,344^{*}$ & 0,052 & 0,173 \\
& & & & 0,063 & 0,000 & 0,009 & 0,021 & 0,384 & 0,160 \\
CN & & & & 1,000 & $0,456^{* *}$ & 0,269 & $0,586^{* *}$ & 0,169 & $0,602^{* *}$ \\
& & & & & 0,003 & 0,059 & 0,000 & 0,166 & 0,000 \\
CNN & & & & & 1,000 & 0,202 & $0,707^{* *}$ & $0,333^{*}$ & $0,501^{* *}$ \\
& & & & & & 0,122 & 0,000 & 0,025 & 0,001 \\
DESINH & & & & & & 1,000 & 0,266 & $-0,051$ & 0,249 \\
& & & & & & & & 0,061 & 0,386 & 0,074 \\
ASERT & & & & & & & 1,000 & 0,269 & $0,533^{* *}$ \\
ICPG & & & & & & & & & 0,059 & 0,000 \\
& & & & & & & & & 1,000 & $0,496^{* *}$ \\
PINFL & & & & & & & & & 0,000 \\
\end{tabular}

**Correlación signif. 0,$01 ; *$ Correlación signif. 0,05

CTOT $=$ Comunicación Total; GT $=$ Comunicación Gestión Tarea; CONC $=$ Comunicación Conceptual; CN= Comunicación Conceptual Novedosa; CNN= Comunicación Conceptual No Novedosa; EMO= Comunicación Socioemocional; DESIN= Comunicación Desinhibida; ASERT= Asertividad; ICPG= Influencia Real (contribución al producto grupal); PINFL= Influencia Percibida

En el caso de los grupos mediados por computadora (CMC) se observó una asociación significativa existente entre la asertividad del discurso y la centralidad comunicacional: 
comunicación total $(r=0,29, \quad p<0,05)$; con la comunicación dirigida a gestionar las interacciones $(r=0,25, p<0,05)$, con la comunicación de tipo conceptual $(r=0,25, \quad p<0,05)$. Dentro de esta categoría, la asertividad se vio asociada únicamente con las alocuciones no novedosas $(r=0,25, \quad p<0,05) . \quad \mathrm{Ni} \quad$ la comunicación socioemocional, ni la desinhibida presentaron asociaciones significativas con la asertividad del discurso $(p>0,05)$

En tanto la influencia (real y percibida) presentó variaciones con relación a la comunicación y la asertividad. Tanto la comunicación como la asertividad estuvieron asociadas en forma baja con la percepción de influencia. Mientras que la influencia real se vio asociada únicamente con las alocuciones novedosas $(p<0,05)$ (Ver Tabla 2).

Tabla 2. Correlación ro de Spearman entre los tipos de comunicación, la asertividad del discurso y la influencia (real y percibida) en grupos CMC. Tarea de Recuperación de Memoria.

\begin{tabular}{|c|c|c|c|c|c|c|c|c|c|c|}
\hline & CTOT & GT & CONC & EMOC & $\mathrm{CN}$ & $\mathrm{CNN}$ & DESIN & ASERT & ICPG & PINFL \\
\hline \multirow[t]{2}{*}{$\overline{\text { CTOT }}$} & 1,000 & $0,599 * *$ & $0,871 * *$ & $0,357 * *$ & $0,280^{*}$ & $0,862^{* *}$ & 0,099 & $0,294^{*}$ & $\begin{array}{c}-0,099 \\
\end{array}$ & $0,337^{*}$ \\
\hline & & 0,000 & 0,000 & 0,008 & 0,031 & 0,000 & 0,258 & 0,025 & 0,258 & 0,012 \\
\hline \multirow[t]{2}{*}{ GT } & & 1,000 & 0,183 & 0,137 & 0,110 & 0,218 & 0,141 & $0,254 *$ & $-0,027$ & $0,300 *$ \\
\hline & & & 0,115 & 0,185 & 0,235 & 0,075 & 0,178 & 0,046 & 0,429 & 0,023 \\
\hline \multirow[t]{2}{*}{$\mathrm{CONC}$} & & & 1,000 & $323 *$ & $0,291 *$ & $0,969 * *$ & $-0,010$ & $0,258^{*}$ & $-0,107$ & $0,306^{*}$ \\
\hline & & & & 0,015 & 0,026 & 0,000 & 0,475 & 0,044 & 0,243 & 0,020 \\
\hline \multirow[t]{2}{*}{ EMOC } & & & & 1,000 & 0,052 & $0,354 * *$ & $0,699 * *$ & $-0,023$ & 0,014 & 0,208 \\
\hline & & & & & 0,366 & 0,009 & 0,000 & 0,441 & 0,464 & 0,085 \\
\hline \multirow[t]{2}{*}{$\mathrm{CN}$} & & & & & 1,000 & 0,125 & $-0,029$ & 0,085 & $0,299 *$ & $0,299 *$ \\
\hline & & & & & & 0,207 & 0,424 & 0,290 & 0,023 & 0,023 \\
\hline \multirow[t]{2}{*}{$\mathrm{CNN}$} & & & & & & 1,000 & 0,019 & $0,256^{*}$ & $-0,148$ & $0,252^{*}$ \\
\hline & & & & & & & 0,452 & 0,045 & 0,165 & 0,048 \\
\hline \multirow[t]{2}{*}{ DESINH } & & & & & & & 1,000 & $-0,094$ & $-0,106$ & $-0,117$ \\
\hline & & & & & & & & 0,269 & 0,243 & 0,221 \\
\hline \multirow[t]{2}{*}{ ASERT } & & & & & & & & 1,000 & 0,149 & $0,459 * *$ \\
\hline & & & & & & & & & 0,164 & 0,001 \\
\hline ICPG & & & & & & & & & 1,000 & $0,359 * *$ \\
\hline PINFL. & & & & & & & & & & $\begin{array}{l}0,008 \\
1,000\end{array}$ \\
\hline
\end{tabular}

**Correlación signif. 0,$01 ; *$ Correlación signif. 0,05.

CTOT = Comunicación Total; GT = Comunicación Gestión Tarea; CONC = Comunicación Conceptual; EMO= Comunicación Socioemocional; $\mathrm{CN}=$ Comunicación Conceptua Novedosa; CNN= Comunicación Conceptual No Novedosa; DESIN.= Comunicación Desinhibida; ASERT = Asertividad; ICPG = Influencia Real (contribución al producto grupal); PINFL.. = Influencia Percibida

En síntesis: tanto en el medio presencial (CAC) como en forma mediada por computadora (CMC) para tareas de recuperación de memoria la asertividad del discurso se vio asociada con la comunicación en general, y con los diferentes tipos de alocuciones. Para ambos medios la comunicación desinhibida no estuvo asociada con la asertividad de los sujetos. La comunicación socioemocional también presentó asociaciones nulas o muy bajas con relación a la asertividad en el discurso de los participantes.

Mientras que, la diferencia principal entre los medios de interacción (cara a cara y mediado por computadora) se vio reflejada en las asociaciones bajas que se presentaron en línea general, en los medios electrónicos a diferencia de los canales presenciales cara a cara.

En cuanto a la influencia de los sujetos (real y percibida) en ambos grupos (cara a cara y mediado por computadoras) la influencia real no estuvo asociada con la asertividad, solamente la percepción que poseían los integrantes de la influencia ejercida se asoció de forma significativa con la asertividad del discurso.

\section{Consideraciones finales}

Los resultados avalan la asociación existente entre la centralidad de la comunicación y la asertividad del discurso, de modo que, quien 
monopoliza la discusión o emite más alocuciones durante las interacciones, a quien posee un discurso más asertivo y es quien influye y es visualizado como sujeto influyente dentro del grupo. La influencia real ejercida en el grupo no presentó asociaciones significativas con la asertividad, para ninguno de los medios (CAC/ CMC).

De todos modos dichas correlaciones son más débiles en los canales mediados por computadora, donde la influencia real no mantiene asociaciones significativas tampoco con la centralidad comunicacional (únicamente en forma débil con las comunicaciones novedosas, lo cual, examinado en forma global no posee demasiada relevancia). Es decir, que los canales mediados electrónicamente no favorecen la correcta visualización de quién influyó al grupo, se les dificulta la correcta percepción del líder que monopolizó la discusión y los resultados.

Se pudo corroborar para ambos medios además, que las comunicaciones de carácter socioemocional y sobre todo la comunicación desinhibida, si bien juegan un papel importante en la dinámica grupal, no ocurre lo mismo con relación a la asertividad o la influencia que se manifiesta en el grupo. Probablemente estas variables desempeñen un rol fundamental en aspectos tales como el clima grupal, pero no con aspectos socio cognitivos como las alocuciones, influencia o asertividad de los participantes.

Este trabajo, si bien permite visualizar con mayor precisión el tipo de comunicación que se da en los pequeños grupos en medios presenciales y mediados por computadora y su relación con la asertividad y la influencia (real y percibida), no agota las discusiones y posteriores investigaciones, sobre todo tendientes a corroborar o refutar los hallazgos aquí reportados, promoviendo la apertura a nuevas líneas de investigación tendientes a comprobar si la naturaleza de la tarea genera diferencias en los aspectos sociocognitivos implicados en los grupos.

Finalmente aclarar que, en este trabajo la asertividad fue evaluada en forma específica y con carácter microsocial, apuntando a la particularidad de la resolución de problemas intelectivos. Entendida como asertividad del discurso, no como habilidades o destrezas sociales, lo cual puede llegar a ser futuro objeto de nuevos trabajos y recategorizaciones a considerar.

\section{Referencias}

Alberti, R. E. (1977). A statement of "principles por ethical practice of assertive behavior training". Em R. E. Alberti (org.). Assertivennes: Innovations. Aplications, Issues. San Luis Obispo, California: Impact.

Alberti, R. E. \& Emmons, M. L. (1978). Your perfect right: A guide to assertive behavior. San Luis Obispo, California: Impact.

Argyle, M. (1981). The nature of social skill. Em M. Argyle (Comp.), Social skills and bealth. Londres: Methuen.

Argyle, M. (1984). Some new developments in social skills training. Bulletin of the British Psychological Society, 53, 405-410.

Arkowitz, H. (1981). Assessment of social skills. Em M. Hersen \& A. S. Bellack (Comps.). Behavioral assessment: a practical handbook $\left(2^{\mathrm{a}}\right.$ ed.). Nova Iorque: Pergamon.

Bales, R. F \& Strodtbeck, F. L. (1951). Phases in group problem-solving. Journal of Abnormal and Social Psychology, 46. 485-495.

Bales, R. F. \& Cohen, S. P. (1979). SYMLOG: $A$ system for the multiple level observation of groups. Nova Iorque: Free.

Bartlett, F. C. (1932). Remembering: a study in experimental and social psychology. Cambridge university.

Bordenave, J. (1985). El proceso de la comunicación. Bs. As: Ateneo.

Borgatti, S. P., Everett, M. G. \& Freeman, L. C. (1999). UCINET 5 for Windows. Software for social network analysis. Natick: analytic technologies.

Caballo, V. E. (1986). Evaluación de las habilidades sociales. Em R. Fernández Ballesteros \& J. A. Carrobles (Comps.), Evaluación conductual: metodología y aplicaciones $\left(3^{\mathrm{a}}\right.$ ed.). Madrid: Pirámide.

Caballo, V. E. (1991). El entrenamiento en habilidades sociales. Em V. E. Caballo (Org.), Terapia y modificación de conducta (p. 403-443). Madrid: Siglo Veintiuno.

Caballo, V. E. (1993). Manual de evaluación y 
entrenamiento de las habilidades sociales. Madrid: Siglo Veintiuno.

Carneiro, R. S. \& Falcone, E. M. (2004). Um estudo das capacidades e deficiências em habilidades sociais na terceira idade. Psicología em Estudo, 9(1), 119-126.

Carrobles Costa Del Sur, B. (1979). La práctica en la terapia de la conducta: teoría y métodos de aplicación para la práctica clínica. Madrid: Pablo del Río.

Carter, L. F. \& Haythorn, W.; Meirowitz, B. \& Lanzetta, J. (1951). The relation of categorizations and ratings in the observation of group behavior. Human Relations, 4. 239254.

Del Prette, Z. A. P. \& Del Prette, A. (1999). Psicologia das Habilidades Sociais: Terapia e educação. Petrópolis: Vozes

Del Prette, Z. A. P. \& Del Prette, A. (2001). Inventário de habilidades sociais: manual de aplicação, apuração e interpretação. São Paulo: Casa do Psicólogo.

Del Prette, Z. A. P., Del Prette, A., Barreto, M. C. M., Bandeira, M., Rios-Saldaña, M. R., Ulian, A. L. A. O., Gerk-Carneiro, E. F., Falcone, E. M. O. \& Villa, M. B. (2004). Habilidades sociais de estudantes de Psicologia: um estudo multicêntrico. Psicologia Reflexão e Crítica, 17(3), 341-350.

Ehuletche, A. \& Terroni, N. (2002). Elaboración de categorías analíticas de la comunicación en interacciones colaborativas. Revista del Instituto de Rosario de Investigaciones en Ciencias de la Educación (IRICE), 16, 185-195.

Emitt, S. \& Gorse, C. (2006). Communication in construction teams. Spon Research.

Falcone, E. O. (1989). A eficácia do tratamento em grupo da ansiedade social. Psicologia Clínica, Pós-Graduação e Pesquisa PUC-RJ, 4, 75-91.

Falcone, E. O. (1995). Grupos. Em B. Rangé (Org.), Psicoterapia comportamental e cognitiva: pesquisa, prática, aplicações e problemas (pp. 159169). Campinas: Editorial Psy.

Falcone, E. O. (1998). A avaliação de um programa de treinamento da empatia. Dissertação de Doutorado, Curso de Pós-Graduação em Psicologia Clínica. Universidade de São Paulo:
São Paulo.

Falcone, E. (2000). Habilidades sociais: para além da assertividade. Em R. C. Wielenska (Org.), Sobre comportamento e cognição: questionando e ampliando a teoria $e$ as intervenções clínicas e em outros contextos, vol. 6 (pp. 211-221). São Paulo: Set.

Falcone, E. M. O., Carneiro, R. S., Chicayban, L. M., Oliveira, M. G. S., Pedrozo, A. L. B. \& Ferreira, M. C. (2001). A construção de um sistema de classificação das habilidades sociais. Em Congresso Iberoamericano de Psicologia Clínica e da Saúde (Org.). Avanços recentes em psicologia clínica e da saúde. Resumo do II Congresso Iberoamericano de Psicologia Clínica e da Saúde (p. 371). Guarujá: APICSA.

Fensterhein, H. \& Baer, J. (1976). No diga sí cuando quiere decir no. Barcelona: Grijalbo.

Fisher, B. A. (1970). Decision emergence: phases in group decision-making. Speech Monograph, 37, 53-66.

Freeman, L. C. (1979) Centrality in social networks: conceptual clarification. Social Networks, 1, 215-239.

Furtado, E. S., Falcone, E. M. O \& Clark, C. (2003). Avaliação do estresse e das habilidades sociais na experiência acadêmica de estudantes de medicina de uma universidade do Rio de Janeiro. Interação (Curitiba), 7(2), 4351 .

Goode, J.; Johnson, M. (1991). Putting out the flames: The etiquette and law of e-mail. Online, Nov. 61-65.

Gouran, D. S. (1969). Variables related to consensus in group discussions of questions of policy. Speech Monographs, 36, 387-391.

Gouran, D. S \& Hirokawa, R. Y. (1983). The role of communication in decision- making groups: A functional perspective. Em M. S. Mander (Org.). Communications in transition (pp.166-185). New York: Praeger.

Güell, M. \& Muñoz, J. (2000). Desconócete a ti mismo. Programa de alfabetización emocional. Barcelona: Paidós.

Harasim, L. M.; Winkelmans, T. (1990). CMC scholarly collaboration. Knowledge: creation, 
diffusion, utilization, 11(4), 382-409.

Hersen, M. \& Bellack, A. (1977). Assessment of Social Skills. Em A. R. Ciminero, A.S. Calhoun \& H. E. Adams (Comp.), Handbook for behavioral assessment. Nova Iorque: Wiley.

Hiltz, R. S., Johnson, K. \& Turoff, M. (1986). Experiments in group decision making: Communication process and outcome in faceto-face versus computerized conferences. Human Communication Research, 13(2), 225-252.

Hirokawa, R. Y. \& Poole, M. S. (1996). Communication and group decision making. London: Sage.

Jakobson, R. (1963). Essays of general linguistics. Paris: Minuit.

Kelly, J. A. (1982). Social skills training: a practical guide for interventions. Nueva York: Springer.

Kiesler, S., Siegel, J. \& McGuire, T. W. (1984). Social psychological aspects of computermediated communication. American Psychologist, 39(10), 1123-1134.

Laughlin, P. R. \& Ellis, A. (1986). Demonstrability and social combination processes on mathematical intellective tasks. Journal of Experimental Social Psychology, 22, 177-189.

Linehan, M. M. (1984). Interpersonal effectiveness in assertive situations. Em E. A. Bleechman (Comp.). Behavior modification with women. Nueva York: Guilford.

Mabry, E. A.(1975). An exploratory analysis of a developmental model for task-oriented small groups. Human Communication Research, 2, 6674.

MacKay, D. (1988). Dificuldades sociais e interpessoais. Em H. Lettner \& B. Rangé (Orgs.), Manual de psicoterapia comportamental (pp. 137-148). São Paulo: Manole.

Mandler, J. M \& Johnson, N. S. (1977). Remembrance of things parsed: Story structure and recall. Cognitive Psychology, 9, 111-151.

Matos, M. G. (1997). Comunicação e gestão de conflitos na escola. Lisboa: FMH.

Navarro Rodríguez, M. (2000). Asertividad y Teoría de decisiones: El rol del orientador escolar.
Durango,

México.

http:/ / wwww.alejandria.com/00/colaboracio nes.htm

Peiró, J. M., Prieto, M. \& Zornoza, A. M. (1993). Nuevas tecnologías telemáticas y trabajo grupal. Una perspectiva psicosocial. Psicothema, 5, 287-305.

Rathus, S. A. (1973). A 30-item schedule for assesing assertiveness. Behavior Therapy, 4, 398406.

Roselli, N., Bruno, M. \& Evangelista, L. (2000). Análisis experimental de la interacción sociocognitiva mediada por sistemas informáticos de intercomunicación escrita. Aplicación al campo educativo. X Congreso Argentino de Psicología: La Psicología en el siglo XXI: vigencia de sus prácticas. Rosario.

Shannon, C. E. \& Weaver, W. (1949). The mathematical theory of communication. Urbana: University of Illinois.

Schroeder, H. E. \& Rakos, R. F. (1983). The identication and assessment of social skills. Em R. Ellis \& D. Whittington (Comps.), New directions in social skill training. Londres: Croom Helm.

Siegel, J., Dubrovsky, V., Kiesler, S. \& McGuire, T. (1986). Group process in computer mediated communication. Organizational behavior and buman decision processes, 37(2), 157-187.

Terroni, N. (2001). Representación de influencia en grupos interactuando en forma presencial y mediada por computadora en tareas de toma de decisión. Informe de Avance de Beca de Perfeccionamiento. Facultad de Psicología, UNMDP. SECyT

Terroni, N. (2003a). La centralidad en las comunicaciones y la percepción de influencia en los pequeños grupos. Psico-USF, 8(1) 3946.

Terroni, N. (2003b). Representación de influencia en grupos interactuando en forma presencial y mediada por computadora en tareas de toma de decisión. Informe de Beca de Perfeccionamiento. SECyT, UNMDP.

Terroni, N. (2005). La asertividad del discurso y la centralidad comunicacional en pequeños 
grupos. Em J .Vivas (Comp.). Las Ciencias del Comportamiento en los albores del siglo XXI. (360371). UNMDP.

Terroni, N. (2006). La comunicación y la percepción de influencia en grupos cara a cara y mediados por computadora. Su relación con la asertividad de la comunicación y la experticia percibida. Informe Final de Beca Doctoral, categoría 2. Facultad de Psicología, UNMDP. Conicet.

Terroni, N. (2007). La comunicación y la percepción de influencia en grupos cara a cara y mediados por computadora. Su relación con la asertividad de la comunicación y la experticia percibida. Tesis Doctoral, Universidad Nacional de San Luis (UNSL).

Terroni, N. (2008). Las comunicaciones desinhibidas durante las interacciones de los pequeños grupos de trabajo. Revista Perspectivas en Psicología, (en prensa).

Vivas, J. \& Terroni, N. (2001). Restricciones en el canal de comunicación y representación de influencia en situación de toma de decisión.
Psico-USF. 6(1), 19-29.

Watzlawick, P., Bavelas, B. \& Jackson D. (1997). Teoría de la Comunicación Humana. España: Herder.

Wolpe, J. (1958). Psychotherapy by reciprocal inbibition. Palo Alto, California: Standford University.

Wolpe, J. \& Lazarus, A. (1966). Behavior therapy techniques: a guide for treatment of neuroses. Nova Iorque: Pergamon.

Zornoza, A., Orengo, V., Salanova, M., Peiro, J. M. \& Prieto, F. (1993). Procesos de status, liderazgo e influencia en la comunicación mediada. Em L. Munduate Jaca \& M. Barón Duque (comp), Psicología del trabajo y de las organizaciones. 101-114. Sevilla: Eudema.

Recebido em janeiro de 2008 Reformulado em novembro de 2008 Aprovado em janeiro de 2009

Sobre a autora:

Nancy Noemí Terroni é psicóloga, mestre em Psicologia Social e doutoranda em psicologia pela Universidad Nacional de San Luis, Argentina; professora da Cátedra Teorías del Aprendizaje da Facultad de Psicologia da Universidad Nacional de Mar del Plata e pesquisadora do grupo de Psicologia Cognitiva y Educacional da faculdade onde atua. 